\title{
A 29-YEAR-OLD PREGNANT WOMAN WITH METASTATIC BREAST CANCER: A CASE REPORT
}

Ranier Colbek Nascimento1, Sabrina Ribas Freitas

${ }^{1}$ Hospital Universitário de Santa Maria, Universidade Federal de Santa Maria - Santa Maria (RS), Brazil.

Pregnancy-associated breast cancer (PABC) is defined as a breast cancer diagnosed during pregnancy, lactation, or in the first postpartum year. PABC is a rare complication that occurs in approximately $0.01 \%$ to $0.03 \%$ of all pregnancies. The difficulty in diagnosis worsens the prognosis. D.G., 29-year-old, female, noted a mass in her right breast in June 2020. One month later with 13+4 weeks' gestation, she presented to the obstetrics emergency with recurrent episodes of lower back pain. She was released home with pain relief and was instructed to realize a mammography due to the presence of a 4-cm mass on physical examination of the right breast. Patient returned 12 days later with severe low back pain, a BIRADS 4C mammography, and multiple liver lesions in total abdomen ultrasound. Core-needle biopsy demonstrated a stage II invasive ductal carcinoma with hormone receptors positive and human epidermal growth factor receptor 2 positive. There is involvement of the axilla and intramammary lymph nodes. Magnetic resonance imaging of the lower back and sacroiliac joint was performed and found multiple lesions suspected of metastasis in the inferior thoracic vertebrae, lumbar vertebrae, sacrum, ilium, and femurs. Computed tomography (CT) of the thorax identified a $2.3 \times 1.8 \mathrm{~cm}$ irregular lesion in the right breast compatible with the primary neoplasm. Chemotherapy was initiated till she was 31 weeks' gestation. After childbirth, she reinitiates chemotherapy. Three months later, the patient has convulsive episodes. Cranial CT was done and found multiple lesions compatible with brain metastasis, so she initiated brain radiotherapy. PABC can present itself as a challenging situation with nonspecific symptoms and at an advanced stage. Therefore, it is important to have the PABC in our list of differential diagnoses in this patient.

Keywords: Breast Cancer; Pregnancy. 